\title{
INTERFACIAL CRACKS EMANATING FROM PARTIALLY DEBONDED SUBSURFACE CIRCULAR ELASTIC INCLUSIONS
}

\author{
P. B. N. Prasad ${ }^{\star}$ and Anthony W. Giammarise ${ }^{* *}$ \\ *Research Engineer, GE Global Research, GEITC, \#122, Phase-2, Hoodi Village, Whitefield Road, \\ Bangalore 560066, INDIA. \\ ${ }^{* *}$ Principal Engineer, GE Transportation Systems, Erie, PA 16531-0001, USA. \\ prasad.pbn@ge.com
}

\begin{abstract}
The problem of a sub-surface circular elastic inclusion with single interfacial debond in plane elasticity is considered in the present work. The sub-surface defect is modeled as elastic circular inclusion lying at some distance from the free surface. The presence of such defects is critical to the life of components subjected to high contact stresses. The question of the presence of free boundary on the stress intensity factors (SIF) of interfacial arc crack under remote uniform stress fields is addressed in this study. By varying the elastic modulus of inclusion, three cases of elastic mismatch are considered: 1) inclusion is hard corresponding to sub-surface rigid inclusion, 2) inclusion is soft corresponding to sub-surface hole, 3) and, inclusion has same material properties as surrounding matrix (equivalent to sub-surface arc crack in elastic half-plane). Half plane is simulated by means of long crack in the vicinity of the circular elastic inclusion so that half-plane boundary conditions are represented locally. Singular integral equation is setup to simulate half-plane boundary. SIF at arc-crack tip closest to the half-plane is evaluated to study its variation as the inclusion approaches half-plane.
\end{abstract}

\section{Introduction}

When components such as rolling bearings are loaded, the contact stresses tend to initiate surface cracks or sub-surface cracks from defects underneath the surface. Depending upon the distance from contact location, cracks may nucleate from sub-surface defects and propagate finally leading to removal of chunks of material, which in turn leads to amplification in contact stresses and further aggravation of damage. Sub-surface defects can be either voids or inclusions or even small cracks that are formed during manufacturing or formed due to external loads.

The problem of a sub-surface defects (voids/cracks/inclusions) has been the object of study for several authors due to its importance in structural integrity and design and also due to difficulty in inspection (inspection procedure is either time consuming and expensive or detection of a sub-surface defect may not be possible by a particular method like eddy current or dye penetrant testing). In fact, page 94 of Timoshenko and Goodier [1] discusses the importance of distance of separation of circular hole at a distance 'd' from half-plane boundary and its effect on stress concentration at the hole boundary nearest to the half-plane. Markenscoff and Dundurs [2] revisited this problem to find out the asymptotic behaviour of hoop stress at the point closest to half-plane boundary as 'd' decreases. When the separation distance 'd' is very small, the hoop stress on the hole boundary tends to infinity as $\approx d^{-0.5}$. It is interesting to note that the hoop stress on the half-plane boundary vanishes in the limit of ' $d$ ' tending to zero. Also, the singular nature of hoop stress on hole boundary resembles that of a crack tip in the limit of ' $d$ ' becoming zero. Wu and Markenscoff [3] examined the problem of two circular holes separated by some distance. They too obtained inverse square root singularity for hoop stress (at points on hole boundaries where the distance between the two holes is minimum) as the ligament between two holes becomes very thin (two holes are very close to each other). Ramakrishna [4] conducted experimental (photoelastic) and semi-analytical investigations of sub-surface circular elastic inclusions (which can be treated as a general case of hole problem discussed before). Thus, above studies highlight the importance of free boundary-defect interactions due to their relevance to design of structural components.

In this work, we examine the role of half-plane on SIF's of an arc crack at the interface of sub-surface circular elastic inclusion. The current work is therefore a generalization to references [1-4]. The objective here is not to study hoop stress variation on the inclusion boundary but to understand the effect of half-plane on SIF variation at arc crack tips. Half plane is simulated by means of a long crack (whose length is much larger than inclusion radius), which in turn is simulated by means of continuously 
distributed dislocation method. This is achieved by setting up singular integral equation to simulate long crack (or half-plane) and numerically solving it by Gauss-Chebyshev method. Half plane is subjected to uniform remote stress fields (tensile stress). Prasad and Simha [5] derived the solution for a single arc crack along the boundary of circular inclusion subjected to either remote stress field or dislocations in an infinite plane (stress potentials are derived by complex variable methods similar to that of Muskhelishvili's [6]). These solutions are used to set up singular integral equations for simulating half-plane. Modified SIF's at arc crack tips are evaluated numerically to study the effect of half-plane.

\section{Subsurface circular elastic inclusion with single debond at the interface}

Figure 1 shows the geometry of the problem that is will be addressed in this work. Young's modulus and Poisson's ratio of inclusion and matrix are $E_{1}, v_{1}$ and $E_{2}, v_{2}$, respectively. For definitions of other parameters, see Prasad and Simha [5].

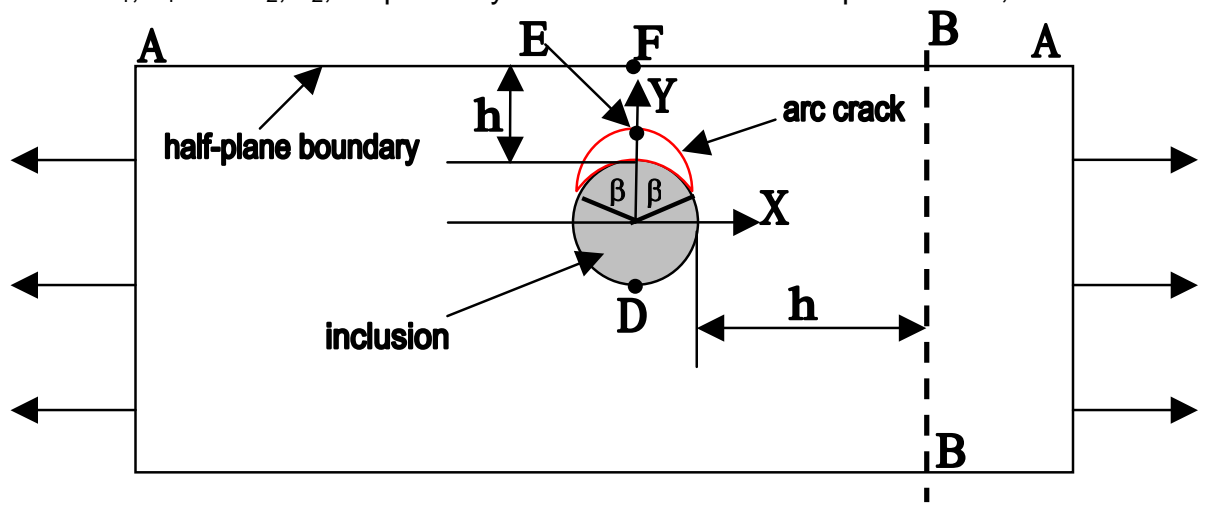

Figure 1. Half plane with a sub-surface debonded circular elastic inclusion at a distance ' $h$ '.

For the inclusion to "see" half-plane (boundary conditions), it is enough to simulate traction free conditions along the line that forms part of half-plane. Of course, care has to be taken to see that the simulated "half-plane crack" is long enough such that no crack-tip interaction effects are felt by circular elastic inclusion. In the current study, "half-plane crack" semi-length is taken as "100a" where "a" is the radius of circular inclusion, to satisfy the above condition. Based on the orientation of simulated "half-plane crack", crack orientation effects (with respect to half-plane) can be studied.

Complex stress potentials for the problem of an arc crack at the interface of circular elastic inclusion and surrounding matrix and subjected to either remote uniform stress field or point force/dislocation are derived by Prasad and Simha [5]. The objective is to cancel tractions acting on potential "half-plane crack" line generated due to remote uniform stresses by means of dislocations. This can be done by means of continuously distributed dislocations technique (see [7] and [8] and references cited therein). By applying this method, singular integral equation for the current problem can be written as,

$$
-\left[\sigma_{n n}(s)+i \sigma_{n \tau}(s)\right]_{\text {farfield }}=\int_{-c}^{c}\left[h_{n}(t)\left(\sigma_{n n}(t, s)+i \sigma_{n \tau}(t, s)\right)+h_{n}(t)\left(\sigma_{\tau \tau}(t, s)+i \sigma_{m}(t, s)\right)\right] d t
$$

where, left hand side denotes the tractions to be cancelled by means of continuously distributed dislocations; "c" denotes half crack length of "half-plane crack"; $h_{n}$ and $h_{\tau}$ are the dislocation densities normal and tangential to "half-plane crack"; $\sigma_{n n}$ and $\sigma_{\mathrm{n}}$ are normal and shear stresses on the "half-plane crack" in local (crack) co-ordinate system; ' $\mathrm{t}$ ' is point of application of dislocation and ' $s$ ' is the point where stresses are evaluated (cancelled). Note that Eq. (1) is written with respect to local coordinate system with origin located at the center of "half-plane crack".

To supplement the above equation, it is necessary to ensure that simulated crack tips are closed and this leads to the following equation:

$$
\int_{-c}^{c}\left[h_{n}(t)+i h_{\tau}(t)\right] d t=0
$$

The method for solving Eq. (1) and (2) are well known (Prasad et al., [7] and, Chen and Hasebe [8] and references cited therein) and are based on Gauss-Chebyshev integration. Finally, SIF at the crack tips of "half-plane crack" are evaluated using Chebyshev-Lagrange interpolation [7,8]. To evaluate SIF at the arc crack tips due to half-plane boundary, following equation is used.

$$
K_{\text {mod }}=K_{\text {farfield }}+\int_{-c}^{c}\left[h_{n}(t) K_{11}(t)+h_{\tau}(t) K_{22}(t)\right] d t
$$

In Eq. (3) above, $\mathrm{K}_{\text {farfield }}$ refers to "complex" SIF $\left(\mathrm{K}_{1}+\mathrm{iK}_{2}\right)$ corresponding to remote uniform stress field whereas, $\mathrm{K}_{11}$ and $\mathrm{K}_{22}$ are due to dislocation densities $h_{n}$ and $h_{\tau}$ [5]; $K_{1}$ and $K_{2}$ are interfacial mode-1 and mode-2 SIF's. Note that all the K's in Eq. (3) are complex and therefore include both mode-1 and mode-2 SIF's. 
In a similar work on slant edge cracks in rails by Hills et al. [9], a different approach is used to simulate slant edge crack from the known solutions of a finite crack subjected to two equal but opposing point forces acting on either faces of the crack in a homogeneous infinite plane and a modified Flamant problem of distributed point forces on the half-plane. An alternating solution procedure was adopted until convergence is met. In the current work, singular integral equation approach is preferred because of the robustness of the procedure and mainly because of the complex boundaries and boundary conditions to be satisfied along inclusion and half-plane boundaries.

In the above numerical scheme, accuracy is ensured to be more than $0.001 \%$ for SIF's computed at the arc crack tips. As the inclusion comes closer to the half-plane boundary, more Gauss points are required to cancel tractions on "half-plane crack" line. Though, the method employed to simulate half-plane is computationally intensive since Gauss-Chebyshev scheme places more points near the crack tips rather than at the center of "half-plane crack" (which is, in fact, the place where we need to cancel tractions; far away from the inclusion, tractions become negligible and hence no need for more points there), other efficient numerical techniques are currently being tried out for faster convergence and better accuracy.

\section{Numerical Results}

Two crack orientations and loadings are considered in current study and SIF's at arc crack tip that is closest to half-plane boundary is computed and plotted in all the results. Of the crack orientations considered are far field normal stress parallel to and perpendicular to arc crack axis. Figure 1 shows arc crack axis ( $y$-axis) normal to applied load and half-plane boundary by section A-A. By creating a different section B-B (see Figure 1) a different half plane configuration is obtained. But, uniform remote load is applied along y-axis (not shown in Figure 1) for this case. Three cases of modulus variations are taken in current study: inclusion is softer (hole), no inclusion (homogeneous) and, inclusion is harder (rigid). Simulations are carried out for arc crack angles up to $60^{\circ}$ as bulk crack closure may commence beyond this arc angle as explained in [5]. An interesting point to note is that bulk crack closure does not necessarily mean crack tip closure and, hence, crack may still grow. But, SIF calculated from the boundary value problem solved is no more valid due to crack face contacts. For the purpose of numerical simulations, Shear modulus and Poisson's ratio of the matrix are taken as $\mu_{2}=2.4 \mathrm{GPa}$ and $v_{2}=0.3$, respectively. Inclusion's shear modulus $\left(\mu_{1}\right)$ is calculated by mismatch parameter $\Gamma=\mu_{2} / \mu_{1}$ and Poisson's ratio is taken as $v_{1}=0.3$. Plane-strain situation is considered for the present analysis. Three values of elastic mismatch are considered: $\Gamma=1 \mathrm{E} 3$ (soft inclusion), $\Gamma=1$ (homogeneous), $\Gamma=1 \mathrm{E}-3$ (rigid inclusion). Actual values of SIF's are plotted against separation distance "h" for increasing arc crack angles $(\beta)$ for crack-load orientations considered in this work.

Figure 2 shows the arc crack-half plane configuration corresponding to section B-B (loading in Y-direction and half-plane is BB) when the inclusion is soft compared to the matrix $(\Gamma=1 \mathrm{E} 3)$. This approximately corresponds to a circular hole beneath the half-plane. It is clear from Figure 2 that SIF's vanish, which is to be expected since arc crack is almost absent (in fact, magnitude of SIF's are very small for this case). Note that the suffix for SIF's in all the figures correspond to arc crack angles (in this work, four arc crack angles are chosen: $10^{\circ}, 30^{\circ}, 45^{\circ}$ and, $60^{\circ}$ ).



Figure 2. SIF vs. distance ' $h$ ' for soft inclusion (hole, $\Gamma=1 \mathrm{E} 3$ ). 
Figure 3 shows the case when there is no mismatch $(\Gamma=1)$ between inclusion and half-plane corresponding to the case of an arc crack below the half-plane. For small arc crack angles $\left(\beta=10^{\circ}\right)$, Mode- 1 shows very little variation for decreasing " $h$ " (slight drop is noticed as " $h$ " becomes small which can't be seen in Figure 3). Change in Mode-1 is insignificant for separation distance " $h$ " above 3 units below which, drop becomes significant for large arc crack angles. For larger values of $\beta$, there is a significant drop in Mode-1 due to crack curvature. It is interesting to note that Mode-1 values for $\beta=30^{\circ}$ and $\beta=45^{\circ}$ are more than for $\beta=10^{\circ}$, showing increased SIF with arc crack length (like a straight crack) for " $\mathrm{h} \geq 1$ ". However, as " $h$ " decreases, Mode1 should also decrease for increasing $\beta$ due to crack curvature effects. On the other hand, Mode-2 SIF increases with decreasing " $h$ ". An interesting observation pertaining to the slope of Mode-2 curves with decreasing " $h$ " for various arc crack angles is that as " $h$ " decreases, the rate of increase (slope) in Mode- 2 increases with increasing $\beta$ for $\beta \leq 45^{0}$ but drops for $\beta=60^{\circ}$ (it is verified from the plot that Mode-2 decreases for "h<0.2"). Note the corresponding change in the slope of Mode-1 (for $\beta=60^{\circ}$ ) for " $\mathrm{h}<0.25$ " (the slope decreases). Hence, for large $\beta$, portion of arc crack faces remote from arc crack tips simulate hole boundary conditions (BC's) thereby changing the local stress field (in the thin ligament joining crack and halfplane). For smaller values of $\beta$, crack tip stress fields dominate. It remains to be explored whether similar SIF behaviour persists as " $h$ " becomes negligibly small. It is conjectured that there might be a competition between singularity due to hole BC's and crack tip singularity interacting with half-plane. The question pertains to which singularity dominates and at what arc crack angle ( $\beta$ ) and separation distance (" $h$ ") for different $\Gamma$.

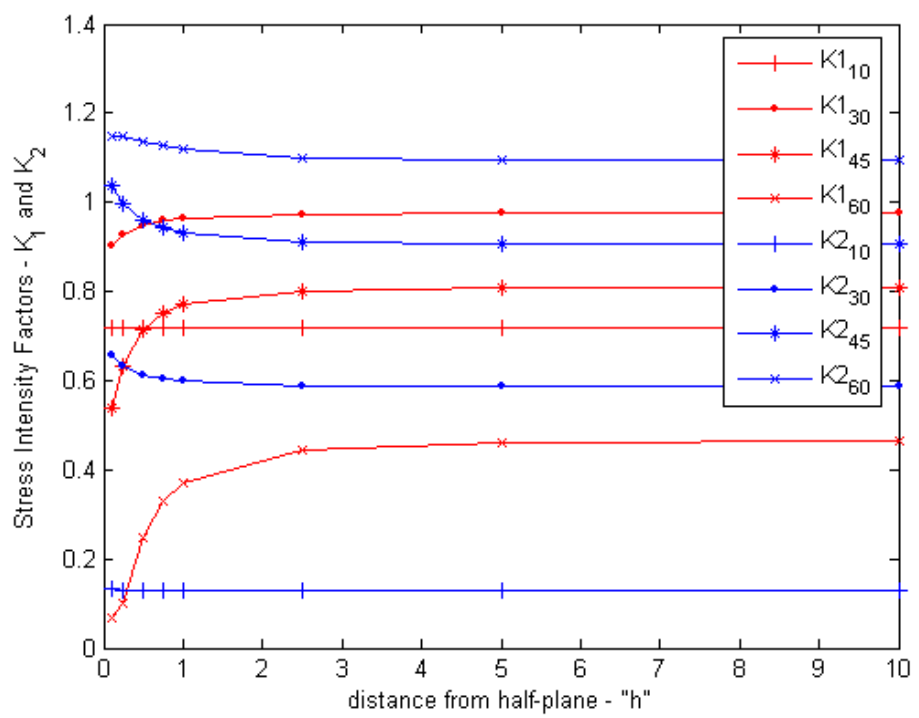

Figure 3. SIF vs. distance ' $h$ ' for no inclusion $(\Gamma=1)$.

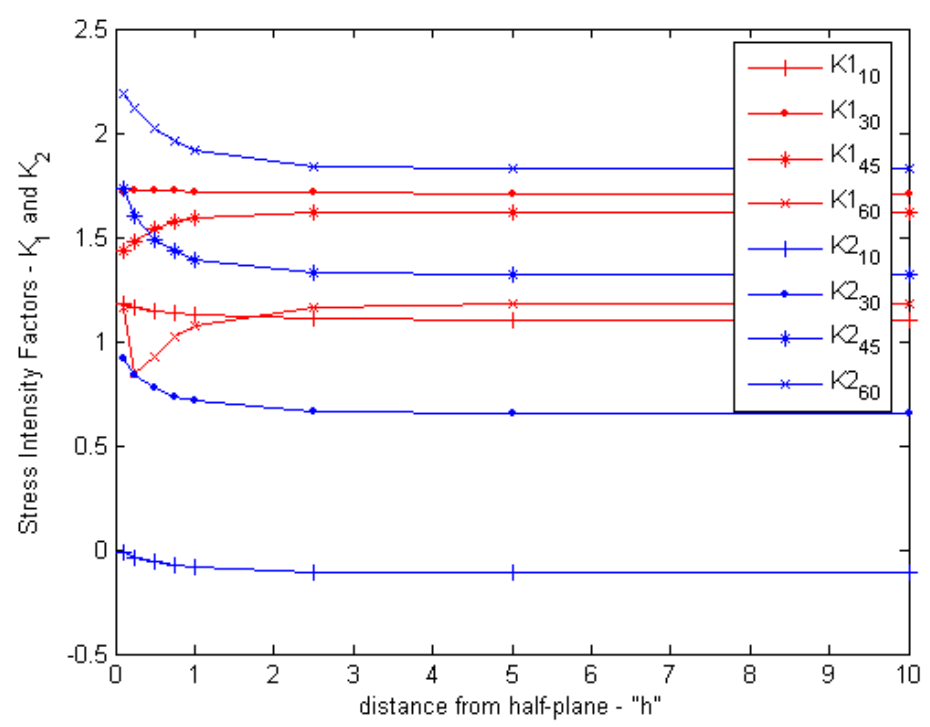

Figure 4. SIF vs. distance ' $h$ ' for hard inclusion $(\Gamma=1 \mathrm{E}-3)$. 
Figure 4 shows the case when inclusion is harder than the half-plane ( $\Gamma=1 \mathrm{E}-3 \approx$ rigid inclusion). For $\beta=10^{\circ}$, Mode- 1 increases with decreasing " $h$ " behaving like a straight crack (for "he<0.1, whether this trend persists for small values of $\beta$ needs to be verified). For $\beta=30^{\circ}$ and $\beta=45^{\circ}$, Mode- 1 decreases with decreasing "h" (though the change for $\beta=30^{\circ}$ is not that significant). However, for $\beta=60^{\circ}$, Mode-1 shows an interesting pattern by decreasing initially and then increasing for decreasing "h". Whether this pattern is retained for "h<<0.1" remains to be explored for all values of $\beta$. Increase in Mode- 1 for $\beta=60^{\circ}$ can be attributed to half-plane - inclusion interaction and the competition between hole effect (from arc crack faces) as well as arc crack tip and thin ligament effects (between inclusion and half-plane) as discussed before (though, it is not clear how the stress fields interact and dominate each other; it is well known [6] that for the case of a rigid inclusion perfectly bonded to the surrounding infinite matrix, the hoop stress is compressive on the inclusion boundary corresponding to point $D$ of Figure 1. When debonding takes place along the inclusion-matrix interface, the evolution of this compressive stress with debonding angle plays a major role in influencing local stress fields. In the present case, half-plane BC's add up to the existing stress fields). Note that Mode-1 increases with increasing $\beta$ (behaving similar to that of straight crack) for " $h>2$ ", below which, halfplane BC's influence their behaviour. For larger values of $\beta$, hole BC's (traction free arc crack faces) should dominate over rigid BC's (zero displacements on bonded portion of the interface). Mode-2 shows a similar trend as seen for the case of $\Gamma=1$ above. The slope of Mode- 2 curves increase for increasing $\beta$ until $\beta=45^{\circ}$ and then decreases for $\beta=60^{\circ}$. A complete stress field analysis will help in understanding the complex interaction issues (work is currently under progress).

The other crack orientation corresponding to section A-A shown in Figure 1 will now be considered. Remote stress is applied along $x$-axis, which is perpendicular to crack axis (y-axis). An important point to note for this crack-load configuration is that for small arc crack angles, the crack is parallel to loading direction and hence no SIF's should result at crack tips. Also, bulk arc crack closure always persists irrespective of arc crack angle (Mode-2 may still exist). However, as crack angle increases, crack tips tend to "open up" inspite of crack faces coming in contact. Thus Mode-I should increase with increasing arc crack angle; however, a different boundary value problem needs to be solved when crack faces are in contact, which is not considered in present study. It is assumed that SIF's computed for arc crack and loading configuration shown in Figure 1 will not differ much from the actual values computed by accounting for bulk arc crack closure.

Figure 5 shows the SIF's for an arc crack at the interface of a soft inclusion (almost a hole). SIF's increase for decreasing "h" but decrease for increasing $\beta$. Figure 5 shows that for small values of $\beta$, SIF increases significantly whereas, for large $\beta$, it increases at a slower rate (again, this trend needs to be verified for " $h<<0.1$ ").

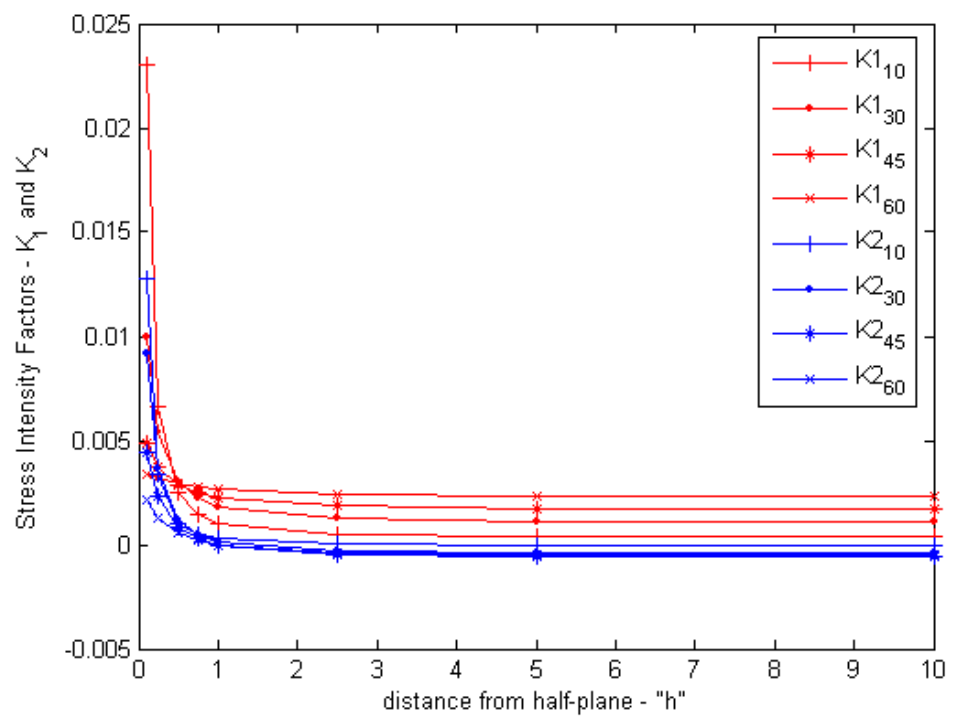

Figure 5. SIF vs. distance ' $h$ ' for soft inclusion (hole, $\Gamma=1 \mathrm{E} 3$ ).

Figure 6 shows SIF's for a subsurface arc crack in a homogeneous half-plane. Mode- 1 for small $\beta\left(10^{\circ}\right.$ and $\left.30^{\circ}\right)$ increases initially and then decreases with decreasing " $h$ ". This indicates that the stress field near the crack tip becomes compressive as " $\mathrm{h}$ " reduces whereas, at the point on the arc crack face closest to half-plane, there is a local stress concentration (tension), which also suggests that at some point over the "outer arc crack face", hoop stress becomes zero between the arc crack tips. With increasing arc crack angle $(\beta)$, Mode-1, however, increases with decreasing " $h$ " (with increasing $\beta$, portion near arc crack tip becomes perpendicular to applied load; hence, the increase). As pointed above, due to bulk crack closure, there may not be a stress concentration (stresses may be compressive) at point $E$ (see Figure 1). This situation is not considered in the present work. On the other hand, Mode- 2 for $\beta=10^{0}$ increases with decreasing " $h$ " while it shows an initial increase and then decrease for $\beta=30^{\circ}$ and $45^{\circ}$. However, for $\beta=60^{\circ}$, Mode- 2 shows a continuously increasing pattern, though, with a decreasing 
slope. It needs to be verified whether this behaviour for large $\beta$ persists for "h<<0.1". For large values of $\beta$, hole BC's (traction free arc crack faces - assuming no crack face contact/bulk closure) dominate in the thin ligament joining half-plane and arc crack. It will be interesting to see the effect of arc crack closure on the "thin ligament stress singularity" with increasing $\beta$ as reported in [2] (work currently in progress).

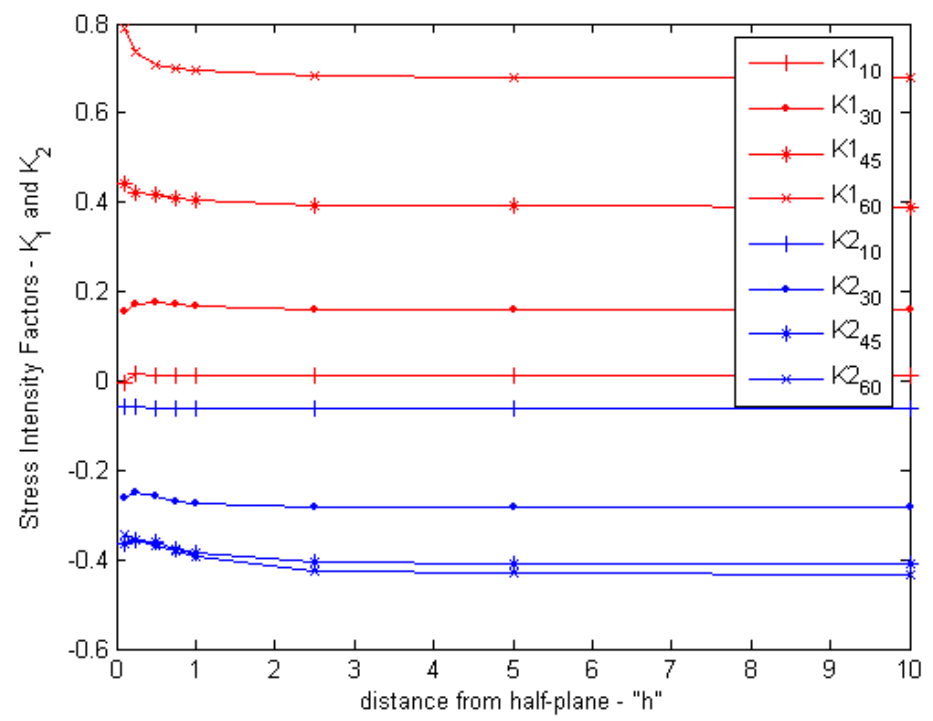

Figure 6. SIF vs. distance ' $h$ ' for no inclusion $(\Gamma=1)$.

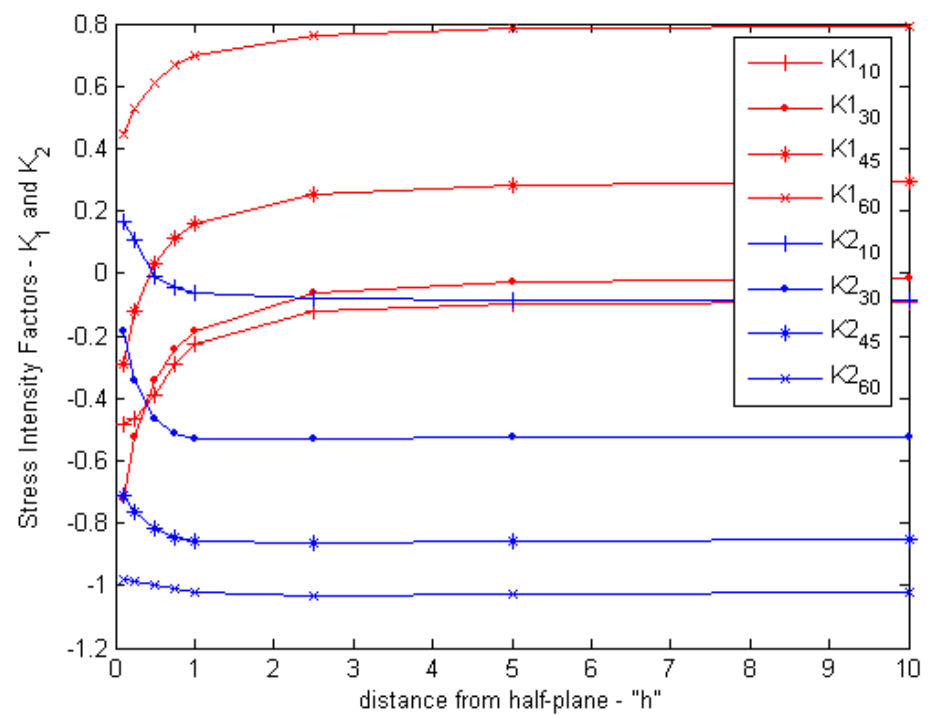

Figure 7. SIF vs. distance 'h' for hard inclusion ( $\Gamma=1 \mathrm{E}-3)$.

Figure 7 considers the case of a hard (rigid) inclusion. The values of Mode- 1 for arc crack angles of $10^{\circ}$ and $30^{\circ}$ can be ignored (since, they are negative). For $\beta=45^{\circ}$ and $60^{\circ}$, Mode-1 decreases with " $h$ " indicating the dominance of rigid $\mathrm{BC}$ 's on the bonded portion of inclusion. In contrast, Mode-2 shows a trend that is opposite of what is shown in Figure 4 (slope increases for $\beta$ upto $30^{\circ}$ and then decreases with increasing $\beta$ for decreasing values of " $h$ "). Most of the subsurface inclusions in components can be treated as rigid and hence this last case gains importance with respect to the question of how a rigid inclusion leads to crack nucleation (for a perfectly bonded rigid inclusion, the stress at the point closest to the half-plane (point $E$ in Figure 1 - when there is no arc crack) is compressive for loading shown in Figure 1 while the stress on the half-plane at point $F$ is tensile. Whether hoop stress at $F$ becomes unbounded or vanishes in the limit of "h" becoming zero needs further analysis; for a subsurface hole, hoop stress at $F$ vanishes as " $h \rightarrow 0$ " [2]). Also, the question of when inclusion debonding occurs vis-à-vis the formation of half-plane edge cracks or inclusion cracking will throw more light on the role of subsurface defects in components failure. 


\section{Conclusions}

The focus of current study is to understand the presence of a subsurface circular elastic inclusion with debond at the interface and subjected to uniform remote fields. The current work is an attempt to model inclusions and voids that are typically formed during manufacturing and relevant issues pertaining to their size and distance from point of application of load. Such parts can fail in several ways under external loading either by cracks nucleating from the defects, or by inclusion cracking, or due to other causes. A typical example could be ball bearings where defects may be far away from the surface when manufactured but due to subsequent wear, these defects come closer to the surface. Generally, subsurface defects are difficult to detect and enough care should be taken during manufacturing to keep the defect sizes below a critical size, which will not lead to crack formation.

An attempt is made in the current work to bring out the complex nature of half plane-defect interactions. Depending on the elastic mismatch between the inclusion and half-plane, the behaviour of SIF's vary. In particular, the variation depends on arc crack angle as discussed before. As crack angle $\beta$ increases, the arc crack portion of the subsurface defect simulates hole $B C$ 's locally which in turn effects crack tip behaviour (and local stress fields). A complete stress analysis will throw more light on such stress field interactions. Also, accounting for bulk arc crack closure (crack face contacts) to evaluate SIF's at the crack tips will help in better predictions of SIF's. Frequently, it is seen in the field that applied loads will either lead to cracking or debonding of inclusion. These cracks grow with loads and fracture the component by removing chunks of material. In this work, an attempt to answer the question: "for a given elastic mismatch and debond angle, which mode of fracture becomes dominant with varying distance from the half-plane under remote uniform stress fields"? is made. A more realistic loading will be to simulate contact conditions (like that between roller and bearing surface) and perform a similar study as done here. Also, the case of two symmetrical arc cracks on the inclusion boundary is under progress to understand multiple crack interactions with the half-plane. An efficient numerical scheme is also being developed to study the above mentioned problems.

Finally, an interesting observation regarding the singular nature of tangential stress, on a subsurface circular hole/cavity, at the point closest to half-plane boundary, as given in [2], is its $\mathrm{h}^{-0.5}$ singularity (where "h" is the thin ligament joining circular hole and half-plane). Similar behaviour for tangential stress on hole boundary in a thin ligament joining two circular holes is given in [3]. It will be interesting to evaluate the nature in which hoop stress becomes unbounded on the face of arc crack at the point that is closest to the half-plane, with respect to arc crack angle as well as elastic mismatch. The question of hoop stress on the inner crack face should also be explored. In the limit, when arc crack angle is large enough (to simulate hole/cavity BC's), this later hoop stress (on inner crack face) should vanish.

\section{Acknowledgments}

Authors would like to thank GE Rail for funding this work.

\section{References}

1. Timoshenko, S. P., Goodier, J. N., Theory of Elasticity, $3^{\text {rd }}$ Edition, McGraw-Hill International (1983).

2. Markenscoff, X., Dundurs, J., "Amplification of Stresses in Thin Ligaments," Int. J. Solids Structures, 29(14/15), 1883-1888 (1992).

3. Wu, L., Markenscoff, X., "Singular Stress Amplification Between Two Holes in Tension," J. of Elasticity, 44, 131-144 (1996).

4. Ramakrishna, D. S., "Stress Field Distortion due to Voids and Inclusions in Contact Problems," PhD Thesis, IISc (1994).

5. Prasad, P. B. N., Simha, K. R. Y., "Interface Crack Around Circular Inclusion: SIF, Kinking, Debonding Energetics," Engng. Frac. Mech., 70, 285-307 (2003).

6. Muskhelishvili, N. I., "Some Basic Problems of the Mathematical Theory of Elasticity," Noordhof, Holland (1954).

7. Prasad, P. B. N., Norio Hasebe., Wang, X. F., "Interaction Between Interfacial Cavity/Crack and Internal Crack - Part II: Simulation," ASME J. Appl. Mech., 72, 394-399 (2005).

8. Chen, Y. Z., Norio Hasebe., "An Alternative Fredholm Integral Equation Approach for Multiple Crack Problem and Multiple Rigid Line Problem in Plane Elasticity," Engng. Frac. Mech., 43, 257-268 (1992).

9. Hills, D. A., Sackfield, A., Uzel, A., "The Green's Function for a Slant Edge Crack," Engng. Frac. Mech., 20(2), 245-253 (1984). 